\title{
On-Demand Separation of Oil-Water Mixtures
}

\author{
Gibum Kwon, Arun. K. Kota, Yongxin Li, Ameya Sohani, Joseph M. Mabry, \\ and Anish Tuteja*
}

With increasing environmental awareness and tighter regulations, novel strategies to separate oils from industrial wastewaters, polluted oceanic waters, and oil-spill mixtures, especially in the presence of surfactants, are highly desired. Membranebased technologies are attractive for demulsification, i.e., the conversion of an emulsion to a free oil-water mixture, because they are relatively energy-efficient and applicable across a wide range of industrial processes. However, demulsification is typically followed by either gravity driven separation or skimming for the complete separation of free oil-water mixtures. ${ }^{[1]}$ In this work, we demonstrate for the first time a gravity driven, membrane-based, single unit operation to separate all types of oil-water mixtures, with $\geq 99.9 \%$ separation efficiency, where the separation can be triggered on-demand, i.e., upon applying an electric field. We envision that our on-demand separation methodology will be useful for a wide range of applications including clean up of oil-spills, fuel-purification, separation of a range of commercial emulsions, waste-water treatment, remote operation of oil-water separation units, microfluidic valves and lab on a chip devices.

An electric field provides a facile route for tuning the wettability of polar (or conducting) liquids. The decrease in the macroscopic contact angle for a sessile polar liquid droplet on a dielectric in response to an electric field is commonly known as electrowetting on a dielectric (EWOD). ${ }^{[2-4]}$ EWOD is expressed by the Young-Lippmann equation as: ${ }^{[5]}$

$\cos \theta^{e w}=\cos \theta+\frac{\varepsilon_{0} \varepsilon_{d}}{2 \gamma_{12} d} V^{2}$

Here, $\theta^{e w}$ is the macroscopic contact angle observed at a distance on the order of dielectric thickness $d$ or larger from the three-phase contact line, ${ }^{[2]} \theta$ is the Young's contact angle ${ }^{[6]} \varepsilon_{0}$ is the vacuum permittivity, $\gamma_{12}$ is the interfacial tension between the liquid and ambient medium, and $V$ is the voltage applied across the dielectric of permittivity $\varepsilon_{d}$. While the macroscopic contact angle decreases under an electric field, the microscopic contact angle close to the three-phase contact line remains unchanged and equal to the Young's contact angle. ${ }^{[7,8]}$ On the other hand, non-polar liquids do not show such a decrease in the macroscopic contact angle.

G. Kwon, Dr. A. K. Kota, Y. Li, A. Sohani, Prof. A. Tuteja Department of Materials Science and Engineering University of Michigan, Ann Arbor, MI 48109, USA E-mail: atuteja@umich.edu

Dr. J. M. Mabry

Space and Missile Propulsion Division

Air Force Research Laboratory, Edwards Air Force Base CA 93524, USA

DOI: 10.1002/adma.201201364
To demonstrate the preferential wettability of water (polar liquid) over oil (non-polar liquid) under an electric field, we conducted EWOD of water (surface tension, $\gamma_{v}=72.1 \mathrm{mN} \mathrm{m}^{-1}$ ) and hexadecane (representative oil, $\gamma_{l v}=27.5 \mathrm{mN} \mathrm{m}^{-1}$ ) on nontextured $50 \mathrm{wt} \%$ fluorodecyl POSS + x-PDMS substrates (solid surface energy, $\gamma_{s v}=10.4 \mathrm{mN} \mathrm{m}^{-1}$ ). Figure $1 \mathrm{a}$ shows the macroscopic contact angles for water and hexadecane as a function of the voltage $V$ applied across the dielectric layer. The macroscopic contact angle for hexadecane $\left(\theta_{\text {hexadecane }}^{\text {ew }}=72^{\circ}\right)$ is independent of $V$ (Figure 1b,c), while the macroscopic contact angle for water decreases from $\theta_{\text {water }}^{\text {ew }}=115^{\circ}$ at $V=0 \mathrm{~V}$ (Figure 1d) with increasing voltage and finally saturates at $\theta_{\text {water }}^{\text {ew }}=56^{\circ}$ for all $V>1.5 \mathrm{kV}$ (Figure 1e). For $\varepsilon_{d}=1.9$, the predictions from the Young-Lippmann equation match well with the experimental data, except for the contact angle saturation at high voltage (Figure 1a). The inability of Eq. 1 to predict contact angle saturation is well documented in literature and continues to remain an active area of research. ${ }^{[2,9]}$

While EWOD is traditionally employed on non-textured substrates, recent studies have conducted EWOD with textured substrates. ${ }^{[10-12]}$ When a liquid contacts a textured substrate, it can adopt either the Cassie-Baxter state ${ }^{[13]}$ or the Wenzel state. ${ }^{[14]}$ On textured substrates, polar liquids can transition from the CassieBaxter state to the Wenzel state in response to the applied electric field. ${ }^{[10-12]}$ In the Cassie-Baxter state, the liquid-air interface makes an angle $\theta$, locally with the substrate. ${ }^{[15-17]}$ As the applied pressure $P_{\text {applied }}$ on the liquid increases, the liquid-air interface bends downward, thereby increasing the sagging angle $\delta \theta$. This in turn causes the liquid-air interface to advance downward along the solid surface to locally maintain an angle $\theta$ with the substrate (Figure 1f). ${ }^{[17]}$ For textured substrates possessing a spherical or cylindrical geometry and sufficiently large pore depth (such that the sagged liquid-air interface does not touch the bottom surface), the liquid-air interface continues to bend and advance downward along the surface curvature with increasing pressure, until it approaches a critical texture angle $\psi_{c r}$ (supporting information (SI) section 1). The critical texture angle corresponds to the location where the liquid-air interface can withstand the maximum pressure $P_{\text {critical }}$, before transitioning to the Wenzel state. For textured substrates possessing a cylindrical geometry, such as the membranes considered in this work, $P_{\text {critical }}$ is given as (see SI section 1$)$ :

$P_{\text {critical }}=\frac{\gamma_{12} \sin \left(\theta-\psi_{c r}\right)}{D+R-R \sin \psi_{c r}}$,

where $\psi_{c r}=\theta-\cos ^{-1}\left(\frac{R \sin \theta}{R+D}\right)$

Here, $R$ is the radius of the cylinder and $D$ is half the intercylinder spacing. 

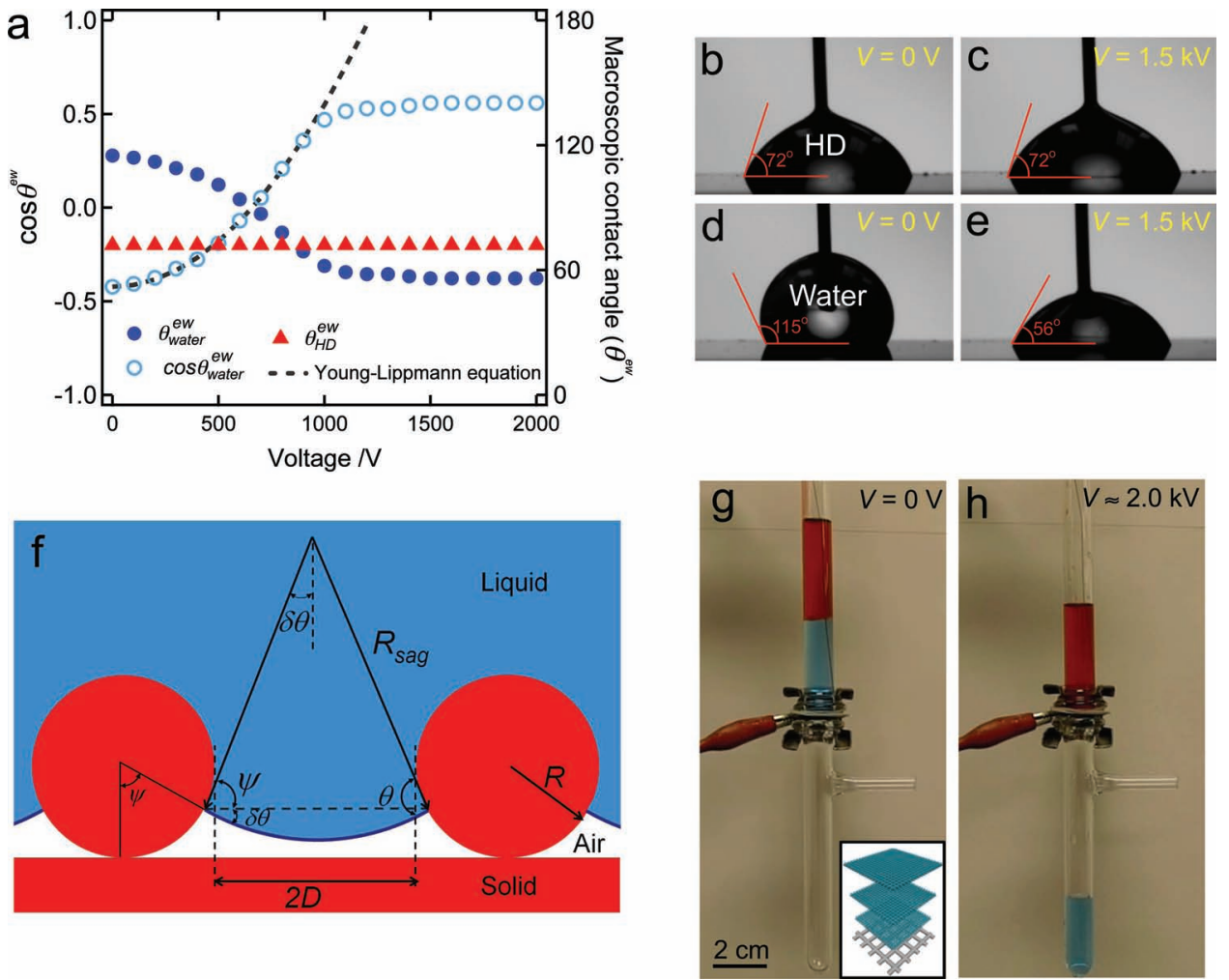

Figure 1. a) Macroscopic contact angles for water and hexadecane (HD) as a function of applied voltage on the non-textured substrate. b,c) The macroscopic contact angle for hexadecane remains unchanged with increasing voltage. d,e) The macroscopic contact angle for water decreases with increasing voltage. f) A schematic showing the pressure-induced sagging of the liquid-air interface. g) An apparatus with a liquid column of free oil (dyed red) and water (dyed blue) above the membrane before applying an electric field. The inset shows a schematic of the membrane module. h) Water permeates through while hexadecane is retained above the membrane when a voltage $V \approx 2.0 \mathrm{kV}$ is applied.

A liquid column of height $h$ above a textured substrate exerts a hydrostatic pressure $P_{\text {hydrostatic }}=\rho g h$ on the liquid-air interface. Here, $\rho$ is the effective density of the liquid column and the $g$ is the acceleration due to gravity. In addition, when a polar liquid is subjected to an electric field, a Maxwell stress $P_{\text {Maxwell }}$ is exerted on the liquid-air interface, pulling it outward along the surface normal. Assuming a configuration similar to a parallel plate capacitor with air as the dielectric medium, ${ }^{[11]}$ $P_{\text {Maxwell }}=\varepsilon_{0} \varepsilon_{d} V^{2} / 2 t_{\text {eff }}^{2}$ Here, $\varepsilon_{d}=1$ is the relative permittivity of the dielectric medium (air), and $t_{e f f}$ is the thickness of the dielectric medium, i.e., the effective distance between the sagged liquid-air interface and the bottom electrode. In EWOD with textured substrates, a polar liquid in the Cassie-Baxter state under gravity, i.e., $P_{\text {hydrostatic }}<P_{\text {critical }}$, will transition to the Wenzel state when the applied pressure $P_{\text {applied }}=\left(P_{\text {hydrostatic }}+\right.$ $P_{\text {Maxwell }}>P_{\text {critical }}$ (SI section 2). On the other hand, a non-polar liquid in the Cassie-Baxter state under gravity, will not transition to the Wenzel state as it will not experience $P_{\text {Maxwell }}$.

For effective on-demand separation of oil-water mixtures, it is essential to use porous oleophobic (or superoleophobic) membranes. Upon applying an electric field, under gravity, such a membrane can allow water to permeate through, but retains the oil (e.g., hexadecane). In previous work, we $\mathrm{e}^{[17-20]}$ and others ${ }^{[21-23]}$ have discussed the critical role of re-entrant texture in the development of superoleophobic surfaces. The oleophobic membrane module (inset in Figure 1g) assembled for this work consists of a stack of three nylon membranes $(2 D=28 \mu \mathrm{m}, R=$ $20.3 \mu \mathrm{m})$ dip-coated with $50 \mathrm{wt} \%$ fluorodecyl POSS + x-PDMS. A stainless steel membrane $(2 D=138 \mu \mathrm{m}, R=56.5 \mu \mathrm{m})$ serves as one electrode, while a copper wire immersed in water serves as the counter electrode. The low solid surface energy and the re-entrant texture of the membrane allow it to support both water $\left(\theta_{\text {water,adv }}^{*}=142^{\circ}\right)$ and hexadecane $\left(\theta_{\text {hexadecane }, a d v}^{*}=99^{\circ}\right)$ in the Cassie-Baxter state before applying an electric field. Here $\theta_{a d v}^{*}$ refers to the apparent advancing contact angle of a liquid on a textured substrate. On non-textured $50 \mathrm{wt} \%$ fluorodecyl POSS $+\mathrm{x}$-PDMS substrates, at $V=0 \mathrm{~V}, \theta_{\text {hexadecane }}=72^{\circ}$ and $\theta_{\text {water }}=$ $115^{\circ}$ (SI section 3). Using Eq. 2, we estimate $P_{\text {critical, hexadecane }}=$ $794 \mathrm{~Pa}$ for the hexadecane-air interface and $P_{\text {critical, } \text { water }}=3540 \mathrm{~Pa}$ for the water-air interface.

The membrane module is sealed between two vertical glass tubes (Figure 1g). A $4 \mathrm{~cm}$ column of water (dyed blue) is added to the upper tube immediately followed by a $4 \mathrm{~cm}$ column of hexadecane (dyed red). The membranes can support the total liquid column because the hydrostatic pressure $P_{\text {hydrostatic }}=694$ $\mathrm{Pa}<P_{\text {critical, }}$ water $=3540 \mathrm{~Pa}$. Under an electric field, the Maxwell stress $P_{\text {Maxwell }}$ increases with increasing voltage $V$ across the membranes. At $V=2.1 \mathrm{kV}$, we calculate $t_{\text {eff }}=81.1 \mu \mathrm{m}$ (SI section 2) and $P_{\text {applied }} \approx P_{\text {critical, water }}$ This matches well with our experimental observation of the permeation of water through 
the membrane module at an applied voltage $V=2.0 \pm 0.3 \mathrm{kV}$. Note that the electric field is applied only for a short period of time (typically a few seconds) because after water permeates through the nylon membranes, contact with the stainless steel membrane creates a path for current flow. After a few minutes, under gravity, all the water is collected in the lower tube, while hexadecane is retained in the upper tube (Figure 1h). The membranes can prevent permeation of the retained hexadecane because the hydrostatic pressure $P_{\text {hydrostatic }}=302 \mathrm{~Pa}$ $<P_{\text {critical, }}$ hexadecane $=794 \mathrm{~Pa}$. A video illustrating the separation of free oil and water is included as SI (Movie S1). To our knowledge, this is the first-ever report of on-demand (electric field actuated) separation of free oil and water driven by gravity. Such on-demand separation can allow for the remote operation and automation of oil-water separation units. Besides oil-water separation, it has other potential applications such as designing microfluidic valves that selectively allow one liquid to flow through while retaining the other. An array of such microfluidic valves would allow for the development of programmable liquid paths ${ }^{[24]}$ with precise control over the position or flux of the desired liquid in a lab on a chip device.

We have also extended our separation methodology to separate oil-in-water emulsions and water-in-oil emulsions with $\geq 99.9 \%$ separation efficiency. Herein, we demonstrate this with two model systems: polysorbate80 (PS80, $0.75 \mathrm{mg} \mathrm{mL}^{-1}$ ) stabilized 50:50 vol:vol hexadecane-in-water emulsions and span80 (1 mg mL ${ }^{-1}$ ) stabilized 30:70 vol:vol water-in-hexadecane emulsions. The dispersed phase size distribution in hexadecanein-water emulsions and water-in-hexadecane emulsions indicates that the highest number fraction of droplet diameters is between 10-20 $\mu \mathrm{m}$ (SI section 4).

To demonstrate the preferential wetting of water over oil, in the presence of PS80, we measured the macroscopic contact angles for water with $1.2 \mathrm{mg} \mathrm{mL}^{-1}$ of PS80 $\left(\gamma_{l_{v}}=40.2 \mathrm{mN} \mathrm{m}^{-1}\right)$ and hexadecane with $0.3 \mathrm{mg} \mathrm{mL}^{-1}$ of PS80 $\left(\gamma_{l v}=24.9 \mathrm{mN} \mathrm{m}^{-1}\right)$ as a function of the voltage $V$ applied across the dielectric layer (Figure 2a). The macroscopic contact angle for hexadecane $\left(\theta_{\text {hexadecane }}^{\text {ew }}=68^{\circ}\right)$ is independent of $V$, while the macroscopic contact angle for water decreases from $\theta_{\text {water }}^{\text {ew }}=80^{\circ}$ at $V=$ $0 \mathrm{~V}$ until it saturates at $\theta_{\text {water }}^{e w}=38^{\circ}$ for all $V>0.8 \mathrm{kV}$. For $\varepsilon_{d}=$ 1.9 (same as in Figure 1a), the predictions from Eq. 1 match well with experimental data. Our experiments also indicate the
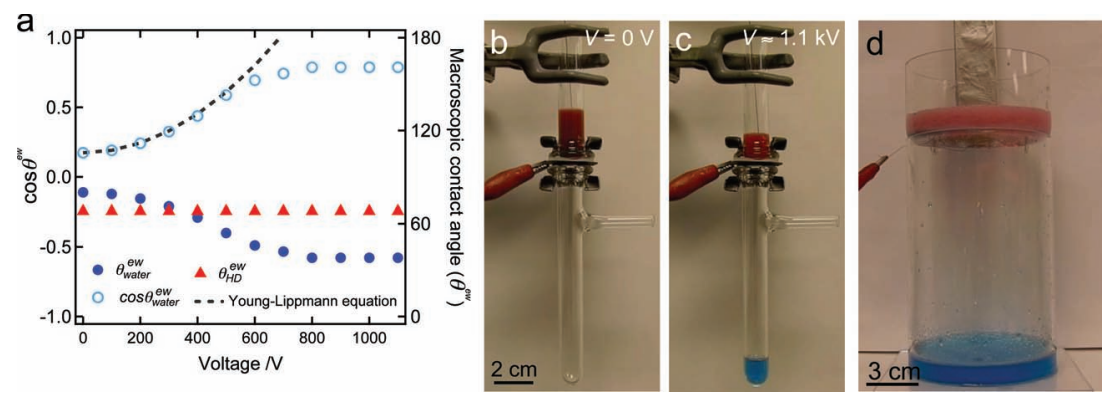

Figure 2. a) Macroscopic contact angles for water and hexadecane (HD) in the presence of PS80 as a function of applied voltage on the non-textured substrate. b) An apparatus with a liquid column of hexadecane-in-water emulsion above the membrane before applying an electric field. c) Water-rich permeate passes through while hexadecane-rich retentate is retained above the membrane when a voltage $V \approx 1.1 \mathrm{kV}$ is applied. d) Separation of the hexadecane-in water emulsion using a scaled-up apparatus. In $(b-d)$ water is dyed blue and hexadecane red. preferential wetting of water over oil, when the oil droplet is surrounded by water, in the presence of PS80, as is the case for oil-in-water emulsions (SI section 5).

The membrane module and the electrode configuration used for the separation of hexadecane-in-water emulsions are the same as those used for free oil and water separation. In the presence of PS80, on non-textured $50 \mathrm{wt} \%$ fluorodecyl POSS + $\mathrm{x}$-PDMS substrates, at $V=0 \mathrm{~V}, \theta_{\text {hexadecane }}=68^{\circ}$ and $\theta_{\text {water }}=79^{\circ}$ (SI section 3). Using Eq. 2, we estimate $P_{\text {critical, hexadecane }}=686 \mathrm{~Pa}$ for the hexadecane-air interface $\left(\gamma_{v}=24.9 \mathrm{mN} \mathrm{m}^{-1}\right)$ and $P_{\text {critical, water }}=$ $1265 \mathrm{~Pa}$ for the water-air interface $\left(\gamma_{v}=40.2 \mathrm{mN} \mathrm{m}^{-1}\right)$. The membranes can support a $3 \mathrm{~cm}$ column of 50:50 vol:vol hexadecane-in-water emulsion (Figure $2 \mathrm{~b}$ ) because $P_{\text {hydrostatic }}=260 \mathrm{~Pa}$ $<P_{\text {critical, water }}=1265 \mathrm{~Pa}$. Upon applying the electric field, $P_{\text {Maxwell }}$ increases with increasing voltage and at $V=1.1 \mathrm{kV}$, we calculate $t_{\text {eff }}=69.7 \mu \mathrm{m}$ (SI section 2), $P_{\text {applied }} \approx P_{\text {critical, water }}$ This matches well with our experimental observation of the permeation of water-rich phase through the membrane module at an applied voltage $V=1.1 \pm 0.3 \mathrm{kV}$. After separation (Figure 2c), the membranes can prevent the permeation of the hexadecane-rich phase (column height $h=1.5 \mathrm{~cm}$ ) because $P_{\text {hydrostatic }}=113 \mathrm{~Pa}<$ $P_{\text {critical, hexadecane }}=686 \mathrm{~Pa}$. Thermogravimetric analysis (TGA) and transmittance measurements indicate that the permeate contains $\sim 0.1 \mathrm{wt} \%$ hexadecane while the retentate contains $\sim 0.1 \mathrm{wt} \%$ water (SI section 6 ). The droplet size distribution in the permeate indicates that virtually all hexadecane droplets exceeding $30 \mu \mathrm{m}$ in diameter are removed (SI section 4). Our analysis also indicates that upon separation the surfactant fractionates into the water-rich and the hexadecane-rich phases depending upon its relative solubility (SI section 7). A video illustrating the separation of hexadecane-in-water emulsion is included as SI (Movie S2). Further, our separation methodology is easy to scale-up. A video illustrating the separation of hexadecane-in-water emulsion using the scaled-up apparatus (Figure 2d) is included as SI (Movie S3).

Unlike oil-in-water emulsions, the separation of water-inoil emulsions occurs through a combination of two processes. First, water-in-oil emulsions demulsify into water-rich and oilrich phases via electrostatic coalescence. ${ }^{[25,26]}$ Immediately after the onset of demulsification, the preferential wettability transition of water over oil under an electric field (similar to the separation of free oil and water) results in complete separation of the water-rich and oil-rich phases.

Similar to PS80, in the presence of span80, the macroscopic contact angle for hexadecane with $1.4 \mathrm{mg} \mathrm{mL}^{-1} \operatorname{span} 80\left(\gamma_{\nu}=25.7 \mathrm{mN} \mathrm{m}^{-1}\right)$, $\theta_{\text {hexadecane }}^{\text {ew }}=68^{\circ}$, is independent of $V$. Since span80 is virtually insoluble in water, the macroscopic contact angles for water as a function of the voltage $V$ applied across the dielectric layer are the same as those shown in Figure 1a.

The membrane module and the electrode configuration for the separation of waterin-hexadecane emulsions are the same as those used for free oil and water, and oilin-water emulsion separations. In the presence of span 80 , on non-textured $50 \mathrm{wt} \%$ fluorodecyl POSS + x-PDMS substrates, at 

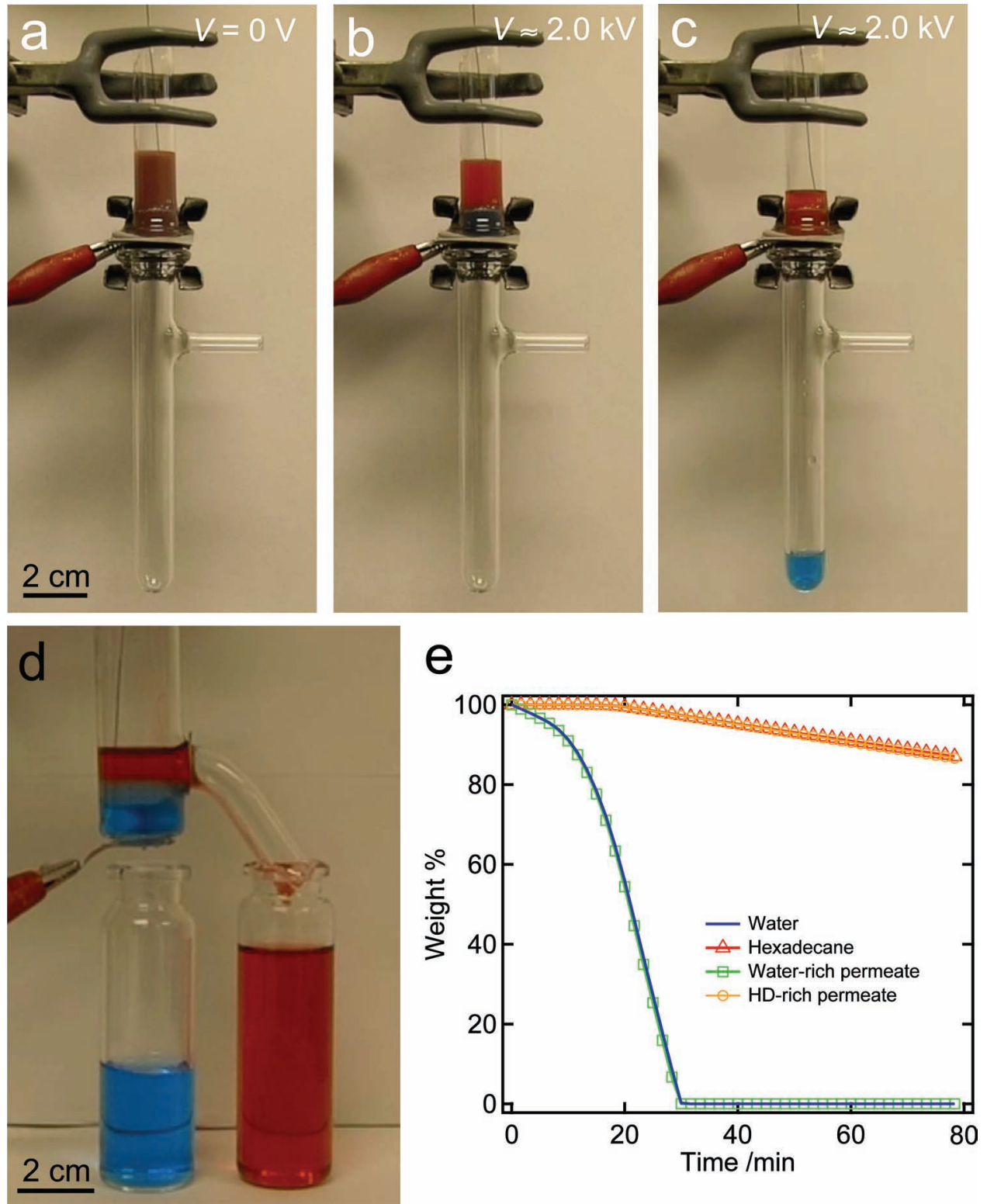

Figure 3. a) An apparatus with a liquid column of water-in-hexadecane emulsion above the membrane before applying an electric field. b) The waterin-hexadecane emulsion is demulsified into the water-rich and the hexadecane-rich phases upon applying an electric field. c) After the onset of demulsification, water-rich permeate passes through while hexadecane-rich retentate is retained above the membrane when a voltage $V \approx 2.0 \mathrm{kV}$ is applied. d) An apparatus used for continuous separation of water-in-hexadecane emulsions. Water-rich permeate passes through the membrane module at the bottom, while hexadecane-rich permeate passes through the hydrophobic and oleophilic membrane on the side-wall. In (a-d) water is dyed blue and hexadecane is dyed red. e) TGA data for the water-rich and hexadecane-rich permeates.

$V=0 \mathrm{~V}, \theta_{\text {hexadecane }}=68^{\circ}$ and $\theta_{\text {water }}=115^{\circ}$ (SI section 3). Using Eq. 2, we estimate $P_{\text {critical, hexadecane }}=708 \mathrm{~Pa}$ for the hexadecaneair interface $\left(\gamma_{l v}=25.7 \mathrm{mN} \mathrm{m}^{-1}\right)$ and $P_{\text {critical, water }}=3540 \mathrm{~Pa}$ for the water-air interface $\left(\gamma_{v}=72.1 \mathrm{mN} \mathrm{m}^{-1}\right)$. The membranes can support a $3 \mathrm{~cm}$ column of 30:70 vol:vol water-in-hexadecane emulsion because $P_{\text {hydrostatic }}=247 \mathrm{~Pa}<P_{\text {critical, hexadecane }}=708 \mathrm{~Pa}$ (Figure 3a). Upon applying the electric field, water-in-hexadecane emulsion demulsifies into water-rich and hexadecane-rich phases due to electrostatic coalescence (Figure 3b). Immediately after the onset of demulsification, $P_{\text {Maxwell }}$ increases with increasing voltage, and at $V=2.2 \mathrm{kV}$, we calculate $t_{\text {eff }}=81.1 \mu \mathrm{m}$
(SI section 2), $P_{\text {applied }} \approx P_{\text {critical, water }}$ This matches well with our experimental observation of the permeation of water-rich phase through the membrane module at an applied voltage $V=2.0 \pm$ $0.3 \mathrm{kV}$. After separation (Figure $3 \mathrm{c}$ ), the membranes can prevent the permeation of the hexadecane-rich phase (column height $h=2.1 \mathrm{~cm}$ ) because $P_{\text {hydrostatic }}=158 \mathrm{~Pa}<P_{\text {critical, hexadecane }}=$ $708 \mathrm{~Pa}$. TGA and transmittance measurements indicate that the permeate contains $\sim 0.1 \mathrm{wt} \%$ hexadecane while the retentate contains $\sim 0.1 \mathrm{wt} \%$ water (SI section 6 ). A video illustrating the separation of water-in-hexadecane emulsion is included as SI (Movie S4). Thus, for the first-time, a single unit operation can 
be used for on-demand separation of free oil-water, oil-in-water emulsions and water-in-oil emulsions driven by gravity.

In the batch separation apparatus discussed above, continuous addition of feed emulsion leads to accumulation of oil above the membrane and oil will eventually permeate through the membrane when $P_{\text {hydrostatic }}>P_{\text {critical, hexadecane. Therefore, }}$ we designed a continuous separation apparatus (Figure 3d) with two types of membrane modules operating in parallelthe membrane module shown in the inset of Figure $1 \mathrm{~g}$ at the bottom, and a hydrophobic and oleophilic membrane $(2 D=$ $37.5 \mu \mathrm{m}, R=12.5 \mu \mathrm{m}, \gamma_{s v}=35.6 \mathrm{mN} \mathrm{m}^{-1}$ ) on the sidewall. The water-in-hexadecane emulsion is continuously fed by a syringe pump. On applying a voltage $V=2.0 \pm 0.3 \mathrm{kV}$, water-rich phase permeates through the membrane module at the bottom, while hexadecane-rich phase permeates through the membrane on the sidewall (Figure 3d). TGA indicates that the water-rich permeate contains $\sim 0.1 \mathrm{wt} \%$ hexadecane and the hexadecane-rich permeate contains $\sim 0.1 \mathrm{wt} \%$ water (Figure $3 \mathrm{e}$ ), which is the limit of detection for the TGA. Karl Fischer analysis indicates that the hexadecane-rich permeate contains $\sim 88 \mathrm{ppm}$ water (SI section 6). Analysis of the hexadecane-rich permeate also indicates that at least $99 \%$ of emulsified water droplets are removed (SI section 8). Flux for the water-rich permeate through the membranes was measured to be $\approx 200 \mathrm{~L} / \mathrm{m}^{2}-\mathrm{hr}$. This value is comparable to those reported in literature for other membranes. ${ }^{[27-32]}$ A video illustrating the continuous separation of water-in-hexadecane emulsion is included as SI (Movie S5).

In summary, we have developed the first-ever membranebased, single unit operation that allows for on-demand separation of various oil-water mixtures using gravity. Using our methodology, we demonstrate the on-demand separation of free oil and water, oil-in-water emulsions and water-in-oil emulsions, with $\geq 99.9 \%$ separation efficiency. The voltage required to trigger the separation was computed using a breakthrough pressure model that incorporates Maxwell stress and the hydrostatic pressure. The predictions from the model were found to match well with our experiments. The ease of scalability of the developed apparatus allowed us to separate larger quantities of oil-water emulsions. Finally, we have also engineered a continuous oil-water emulsion separation apparatus that is triggered on-demand and removes $>99 \%$ of the emulsified droplets.

\section{Experimental Section}

Non-textured substrate fabrication: A 10:1 w:w ratio PDMS mixture of Sylgard184 base:curing agent (Dow Corning) was prepared in a petri dish and cross-linked at $70^{\circ} \mathrm{C}$ for $3 \mathrm{~h} .1 \mathrm{H}, 1 \mathrm{H}, 2 \mathrm{H}, 2 \mathrm{H}$-Heptadecafluorodecyl polyhedral oligomeric silsequioxane (fluorodecyl POSS) was synthesized as described elsewhere. ${ }^{[33]}$ The cross-linked PDMS (x-PDMS) sheet (thickness, $d \sim 120 \mu \mathrm{m}$ ) was sliced into $2 \mathrm{~cm} \times 2 \mathrm{~cm}$ pieces and spincoated with $10 \mathrm{mg} \mathrm{mL}^{-1}$ solutions of $50 \mathrm{wt} \%$ fluorodecyl POSS + PDMS in Asahiklin AK-225 (Structure Probe, Inc) at 1000 RPM for $20 \mathrm{~s}$ and cross-linked at $70{ }^{\circ} \mathrm{C}$ for $1 \mathrm{~h}$. We estimated the solid surface energies using the Owens-Wendt approach. ${ }^{[34]}$

Textured substrate fabrication: $2 \mathrm{~cm} \times 2 \mathrm{~cm}$ pieces of nylon membranes (mesh size $376, R=20.3 \mu \mathrm{m}, 2 D=28 \mu \mathrm{m}$ ) were dip-coated with $10 \mathrm{mg} \mathrm{mL}^{-1}$ solutions of $50 \mathrm{wt} \%$ fluorodecyl POSS + PDMS in Asahiklin AK-225 for $30 \mathrm{~min}$. Subsequently, the membranes were dried at room temperature for $3 \mathrm{~min}$ and cross-linked at $70{ }^{\circ} \mathrm{C}$ for $1 \mathrm{~h}$. The re-entrant texture of the nylon membrane and the low solid surface energy of the
50 wt\% fluorodecyl POSS + PDMS blend coating $\left(\gamma_{s v}=10.4 \mathrm{mN} \mathrm{m}^{-1}\right)$ allow our membranes to support both water and hexadecane in the Cassie-Baxter state before applying an electric field. The hydrophobic and oleophilic membranes used in continuous separation were made by dip-coating stainless steel membranes $(R=12.5 \mu \mathrm{m}, 2 D=37.5 \mu \mathrm{m})$ in $10 \mathrm{mg} \mathrm{mL}^{-1}$ solution of Desmopan 9370 (Bayer Materials Science) in THF.

Contact angle measurement: All contact angles were measured by advancing or receding about $2 \mu \mathrm{L}$ of liquid onto the surface (Ramé-Hart 200-F1 goniometer).

Oil-water emulsions: The formation of an oil-in-water or a water-in-oil emulsion is primarily determined by the hydrophilic-lipophilic balance (HLB) of the surfactant. Hexadecane-in-water emulsions were prepared using polysorbate $80(H L B=15)$, while water-in-hexadecane emulsions were prepared using span $80(H L B=4.3)$. The emulsions were prepared by mixing water, hexadecane and the surfactant using a stir bar (at 700 1200 RPM). We verified whether an emulsion is hexadecane-in-water or water-in-hexadecane by measuring the electrical resistance with a multimeter. Over time, a small degree of demulsification was observed for some emulsions.

\section{Supporting Information}

Supporting Information is available from the Wiley Online Library or from the author.

\section{Acknowledgements}

GK and AKK contributed equally to this work. We thank Dr. Charles Y-C. Lee and the Air Force Office of Scientific Research (AFOSR) for financial support under grants FA9550-10-1-0523 and LRIR-92PLOCOR. We also thank the donors of the American Chemical Society Petroleum Research Fund. We thank Prof. Frieder Mugele at the University of Twente for comments and discussion. We also thank Prof. Nicholas A. Kotov at the University of Michigan for use of facilities. We thank Mr. Brett Wight at the Air Force Research Laboratory for help with Karl Fischer analysis.

Received: April 3, 2012

Published online: June 12, 2012

[1] A. Hong, A. G. Fane, R. Burford, J. Membrane Sci. 2003, 222, 19.

[2] F. Mugele, J. C. Baret, J. Phys.-Condens. Mat. 2005, 17, R705.

[3] R. Shamai, D. Andelman, B. Berge, R. Hayes, Soft Matter 2008, 4, 38.

[4] S. L. Gras, T. Mahmud, G. Rosengarten, A. Mitchell, K. Kalantar-Zadeh, ChemPhysChem 2007, 8, 2036.

[5] B. Berge, CR Acad. Sci. II 1993, 317, 157.

[6] T. Young, Philos. Trans. R. Soc. London 1805, 95, 65.

[7] J. Buehrle, S. Herminghaus, F. Mugele, Phys. Rev. Lett. 2003, 91.

[8] F. Mugele, J. Buehrle, J. Phys.-Condens. Mat. 2007, 19.

[9] D. Klarman, D. Andelman, M. Urbakh, Langmuir 2011, 27, 6031.

[10] G. Manukyan, J. M. Oh, D. van den Ende, R. G. H. Lammertink, F. Mugele, Phys. Rev. Lett. 2011, 106.

[11] J. M. Oh, G. Manukyan, D. van den Ende, F. Mugele, Europhys. Lett. 2011, 93.

[12] T. N. Krupenkin, J. A. Taylor, E. N. Wang, P. Kolodner, M. Hodes, T. R. Salamon, Langmuir 2007, 23, 9128.

[13] A. B. D. Cassie, S. Baxter, T. Faraday Soc. 1944, 40, 0546.

[14] R. N. Wenzel, Ind. Eng. Chem. 1936, 28, 988.

[15] S. Herminghaus, Europhys. Lett. 2007, 79.

[16] A. Marmur, Langmuir 2003, 19, 8343.

[17] A. Tuteja, W. Choi, J. M. Mabry, G. H. McKinley, R. E. Cohen, Proc. Natl. Acad. Sci. USA 2008, 105, 18200. 
[18] W. Choi, A. Tuteja, S. Chhatre, J. M. Mabry, R. E. Cohen, G. H. McKinley, Adv. Mater. 2009, 21, 2190.

[19] A. K. Kota, G. Kwon, W. Choi, J. M. Mabry, A. Tuteja, Nature 2012, DOI 10.1038/nature11201.

[20] A. Tuteja, W. Choi, M. Ma, J. M. Mabry, S. A. Mazzella, G. C. Rutledge, G. H. McKinley, R. E. Cohen, Science 2007, 318, 1618.

[21] A. Ahuja, J. A. Taylor, V. Lifton, A. A. Sidorenko, T. R. Salamon, E. J. Lobaton, P. Kolodner, T. N. Krupenkin, Langmuir 2008, 24, 9.

[22] L. Cao, T. P. Price, M. Weiss, D. Gao, Langmuir 2008, 24, 1640.

[23] A. Marmur, Langmuir 2008, 24, 7573.

[24] C. Quilliet, B. Berge, Curr. Opin. Colloid In. 2001, 6, 34.

[25] M. Elektorowicz, S. Habibi, R. Chifrina, J. Colloid Interf. Sci. 2006, 295, 535.
[26] J. S. Eow, M. Ghadiri, Colloid. Surface. A 2003, 219, 253.

[27] T. C. Arnot, R. W. Field, A. B. Koltuniewicz, J. Membrane Sci. 2000, 169, 1.

[28] A. B. Koltuniewicz, R. W. Field, T. C. Arnot, J. Membrane Sci. 1995, 102, 193.

[29] M. Hlavacek, J. Membrane Sci. 1995, 102, 1.

[30] D. Z. Sun, X. D. Duan, W. X. Li, D. Zhou, J. Membrane Sci. 1998, 146,65 .

[31] A. Maartens, E. P. Jacobs, P. Swart, J. Membrane Sci. 2002, 209, 81.

[32] B. Hu, K. Scott, J. Membrane Sci. 2007, 294, 30.

[33] J. M. Mabry, A. Vij, S. T. Iacono, B. D. Viers, Angew. Chem. Int. Ed. 2008, 47, 4137

[34] D. K. Owens, R. C. Wendt, J. Appl. Polym. Sci. 1969, 13, 1741. 